\title{
水杨酸和精氨酸改性二氧化钛颗粒的制备及其 在油-水界面的光催化反应
}

\author{
李 䂞 ${ }^{1}$, 张巧玲 ${ }^{1}$, 范红蕾 ${ }^{1}$, 刘有智 ${ }^{1}$, 魏 水 $^{1}$, 冯玉杰 ${ }^{2}$
}

(1. 中北大学 超重力化工过程山西省重点实验室, 太原 $030051 ; 2$. 哈尔滨工业大学 城市水资源与水环境国家重点 实验室, 哈尔滨 150090)

摘 要: 采用浸渍改性法将水杨酸与精氨酸同时接枝到纳米 $\mathrm{TiO}_{2}$ 表面制备了水杨酸和精氨酸共改性二氧化钛颗粒 $\left(\mathrm{TiO}_{2}-\mathrm{SA} / \mathrm{Arg}\right)$, 并采用傅里叶红外光谱(FT-IR)、X 射线光电子能谱(XPS)、热失重分析(TGA)、紫外-可见光漫反射光 谱(UV-Vis DRS)、扫描电子显微镜(SEM)、高倍透射电子显微(HRTEM)、表面接触角测试及粒度分布分析等技术对 材料的形貌、结构及性能进行表征，同时研究了催化剂对硝基苯的吸附性能及在油-水界面的吸附能力。结果表明: 水 杨酸和精氨酸分别以鳌合和桥连结构稳定修饰到 $\mathrm{TiO}_{2}$ 表面，与未改性 $\mathrm{TiO}_{2}$ 相比，水杨酸和精氨酸共改性 $\mathrm{TiO}_{2}$ 疏水性 及分散性更好, 对硝基苯的吸附能力更强, 并可在油-水界面稳定吸附形成 $\mathrm{O} / \mathrm{W}$ 型 Pickering 乳液。研究了 Pickeing 乳液光催化体系对硝基苯的去除能力，与悬浮体系相比，改性颗粒稳定的 Pickering 乳液体系对高浓度硝基苯去除效 率较高。

关＼cjkstart键＼cjkstart词: 表面改性; 表面结构; 光催化; Pickering 乳液

中图分类号: TQ174 文献标识码: A

\section{Titanium Dioxide Particles Modified by Salicylic Acid and Arginine and Their Photocatalytic Reaction on Oil-Water Interface}

\author{
LI Lei ${ }^{1}$, ZHANG Qiao-Ling ${ }^{1}$, FAN Hong-Lei ${ }^{1}$, LIU You-Zhi ${ }^{1}$, WEI Bing ${ }^{1}$, FENG Yu-Jie ${ }^{2}$
}

(1. Shanxi Province Key Laboratory of Higee-Oriented Chemical Engineering, North University of China, Taiyuan 030051, China; 2. State Key Laboratory of Urban Water Resource and Environment, Harbin Institute of Technology, Harbin 150090, China)

\begin{abstract}
Salicylate acid and arginine-modified $\mathrm{TiO}_{2}$ particles $\left(\mathrm{TiO}_{2}-\mathrm{SA} / \mathrm{Arg}\right)$ were successfully fabricated by impregnation approach using salicylate acid and arginine grafted onto the surface of nano- $\mathrm{TiO}_{2}$. The morphology, structure and properties of modified samples were characterized by means of Fourier-transform infrared spectroscope (FT-IR), X-ray photoelectron spectroscope (XPS), thermogravimetry ansysis (TGA), ultraviolet-visible diffuse reflectance spectroscope (UV-Vis DRS), scanning electron microscope (SEM), high-resolution transmission electron microscope (HRTEM), surface contact angle analysis, and particle size distribution analysis. The nitrobenzene adsorption capacity and the ability to be attracted to the oil-water interface of the modified samples were also confirmed. Results revealed that salicylate acid and arginine were modified to $\mathrm{TiO}_{2}$ surface by chelation and bridging structure, respectively. The modified $\mathrm{TiO}_{2}$ had better hydrophobicity, dispersion properties and adsorption capacity of nitrobenzene than the unmodified $\mathrm{TiO}_{2}$. A stable $\mathrm{O} / \mathrm{W}$ Pickering emulsion was successfully fabricated via the
\end{abstract}

收稿日期: 2015-09-09; 收到修改稿日期：2015-11-17

基金项目: 城市水资源与水环境国家重点实验室开放基金(QA201401)；山西省研究生优秀创新项目(2014301) Open Project of State Key Laboratory of Urban Water Resource and Environment (QA201401); Outstanding Graduate Innovation Project of Shanxi Province(2014301)

作者简介：李 否(1991-), 男，硕士研究生. E-mail: flytolilei@126.com.cn

通讯作者: 张巧玲，副教授. E-mail: zhangqiaoling@nuc.edu.cn 
photocatalyst stably adsorbed to oil-water interface. Nitrobenzene in Pickeing emulsion-based photocatalytic reaction system, stabilized by modified photocatalyst, showed a higher removal rate of high concentrations of nitrobenzene than the suspension system.

Key words: surface modification; surface structure; photocatalysis; Pickering emulsion

纳米 $\mathrm{TiO}_{2}$ 具有化学稳定性好、催化活性强、廉 价无毒以及矿化程度较高等特点, 其光催化性能优 于 $\mathrm{ZnO} 、 \mathrm{WO} 、 \mathrm{CdS}$ 等光催化剂 ${ }^{[1-3]}$, 在紫外光下可以 迅速降解 3000 多种难以降解的有机化合物 ${ }^{[4]}$, 在处 理高浓度、难降解有机废水方面具有独特优势。研 究发现, 高浓度有机废水常以油/水分层形式存在, 纳米 $\mathrm{TiO}_{2}$ 具有表面强亲水特性, 易分散于水相中, 导致污染物与催化剂接触不充分, 传质效率受到一 定限制 ${ }^{[5]}$ 。

20 世纪初, Ramsden ${ }^{[6]}$ 和 Pickering ${ }^{[7]}$ 发现微米级 尺寸的固体粒子可以吸附于油-水界面形成稳定的 乳液, 这种乳液被称为 Pickering 乳液。固体粒子有 效地稳定 Pickering 乳液的前提条件是能够被油/水相 部分润湿。其稳定机制主要有机械阻隔机制、桥连 机理以及三维粘弹粒子网络机理, 即固体粒子形成 单层或多层的紧密固体膜, 阻止液滴聚并, 并形成 三维网状结构导致连续相粘度增加, 降低乳液液滴 迁移速率和程度, 阻止乳液液滴的聚结 ${ }^{[8-9]}$ 。Pickering 乳液乳化剂的用量较少、毒性小、稳定性好, 在化工、 材料、造纸等领域均有着广泛的应用 ${ }^{[10-11]}$ 。将光催化 技术与 Pickering 乳液联合运用, 构建的界面催化体 系可增大污染物与催化剂的接触面积, 在处理有机 污染物特别是高浓度有机物方面显现出独特优势。 改性 $\mathrm{ZnO}^{[12]}$ 、层状六铌酸 ${ }^{[13]}$ 、改性 $\mathrm{TiO}_{2}{ }^{[5]}$ 、负载磷 酸银的碳纳米管 ${ }^{[14-15]}$ 等固体粒子稳定的乳液已用于 光催化降解有机废水, 并取得较好效果。但大部分光 催化剂具有强亲水特性, 不能有效吸附于油/水界面 形成 Pickering 乳液体系, 需要进行表面亲油改性。

近年来, 采用表面活性剂 ${ }^{[16]}$ 、硅烷偶联剂 ${ }^{[17]}$ 、高 分子聚合物 ${ }^{[18]}$ 等有机物对光催化剂进行表面改性可 以降低表面亲水性、扩展吸收光波长、增强对目标 物吸附能力以及降低电子复合率等 ${ }^{[15]}$, 但由于光生 电子-空穴对产生的强氧化性的自由基会直接攻击 表面改性基团, 导致其发生自降解 ${ }^{[19]}$, 如酒石酸、柠 檬酸、乳酸 ${ }^{[20]}$ 等均为牺牲性改性剂, 催化剂无法重复 利用。部分有机酸, 如乙酸 ${ }^{[21]}$ 、水杨酸(SA)及其衍生 物 ${ }^{[22-23]}$ 、精氨酸 $(\mathrm{Arg})^{[24]}$ 、天冬氨酸 ${ }^{[25]}$ 可与 $\mathrm{TiO}_{2}$ 结合 形成紫外光下稳定的螯合或桥连结构, 并显著提升 光催化效率, 但这类物质分子链较短且吸附量低, 对光催化剂表面亲油性改善程度较小。研究发现, 二
氧化钛表面具有多种酸位点(Brønsted 酸位点和 Lewis 酸位点 $)^{[19]}$, 可进行多酸类物质共改性 ${ }^{[26]}$, 进 一步提升催化剂在油-水界面的吸附能力。此外, 学 者还发现 $\mathrm{Arg}$ 改性可以增强光催化剂的光致还原能 力, 在处理难以氧化降解的污染物上具有良好应用 前景 ${ }^{[23]}$ 。基于此，本研究采用 SA 和 Arg 同时对纳米 $\mathrm{TiO}_{2}$ 进行改性, 并将改性颗粒分散于油/水体系形成 Pickering 乳液, 最后将稳定的 Pickering 乳液用于光 催化降解高浓度硝基苯实验研究。

\section{1 实验方法}

\section{1 主要试剂}

纳米 $\mathrm{TiO}_{2}(\mathrm{P} 25$, 德国 Degussa 公司); 水杨酸 $(\mathrm{SA})$ 、 L-精氨酸(L-Arg)、无水乙醇、硝基苯(NB) (分析纯, 国 药集团化学试剂有限公司); 硫酸、氢氧化钠(分析纯, 中国天津北辰方正试剂厂); 实验用水为去离子水。

\section{2 表面改性过程}

配置饱和 SA 水溶液 $20 \mathrm{~mL}(0.0157 \mathrm{~mol} / \mathrm{L})$ 和饱和 L-Arg 水溶液 $10 \mathrm{~mL}(0.854 \mathrm{~mol} / \mathrm{L})$, 混合后, 加入 $4 \mathrm{~g}$ 纳米 $\mathrm{TiO}_{2}$, 置于 $25{ }^{\circ} \mathrm{C}$ 的超级恒温水浴 $(\mathrm{CH} 1020 \mathrm{~T}$ 型, 温控精度 $\pm 0.05{ }^{\circ} \mathrm{C}$, 上海方瑞仪器有限公司)中, 将 超声细胞粉碎机(SCIENTZ-IID 型, 宁波新芝生物科 技股份有限公司) 激发杆置入反应器, 在 $21 \mathrm{~Hz} 、 950 \mathrm{~W}$ 超声条件下反应 $30 \mathrm{~min}$, 然后静置 $24 \mathrm{~h}$ 使得 $\mathrm{TiO}_{2}$ 表 面吸附饱和，真空过滤并采用去离子水 3 次洗涤以 确保纳米粒子表面无游离酸, 冷冻干燥 $48 \mathrm{~h}$, 得到表 面呈淡黄色的固体粉末。

\section{3 颗粒对硝基苯吸附实验}

分别将 $50 \mathrm{~mL}$ 初始浓度从 $5 \mathrm{mg} / \mathrm{L}$ 至 $100 \mathrm{mg} / \mathrm{L}$ 的 NB 溶液放置于 $100 \mathrm{~mL}$ 具塞雉形瓶中, 再分别加 入 $1 \mathrm{mg}$ 的纳米颗粒, 置于恒温振荡器中, 调节温度 为 $25{ }^{\circ} \mathrm{C}$, 振荡 $24 \mathrm{~h}$ 后, 用高效液相色谱仪(HPLC, UltiMate3000, 美国赛默飞公司)测量瓶中的溶液 NB 浓度。

\section{4 颗粒在油/水界面的吸附及 Pickering 乳液 的制备}

油相为 $\mathrm{NB}$, 固体颗粒质量分数为 $2.5 \%$, 超声 $(21 \mathrm{~Hz} 、 950 \mathrm{~W})$ 乳化 $10 \mathrm{~min}$, 配置油水体积比为 $1: 4$ 、 
1:3 和 1:2 三组 Pickering 乳液, 随即在确定的时间内计 量 Pickering 乳液分层高度, 并用光学显微镜(SMZDV320, 重庆奥特仪器有限公司)记录乳液微观形态。

\section{5 光催化降解硝基苯实验}

以 NB 降解效率来评价体系的光催化性能, 采用 $500 \mathrm{~W}$ 氙灯为光源和具有夹套的圆柱形玻璃反应器 作为反应装置, 在反应器和光源之间插入隔热片滤 去红外光部分, 以消除热效应的影响, 在光催化反 应过程中往夹套中通入冷凝水, 反应器中液面与光 源之间的垂直距离为 $35 \mathrm{~cm}$ 。配置 $80 \mathrm{~mL}$ 油相体积分 数为 $10 \%$ 的 $\mathrm{NB} /$ 水混合液, 加入 $2.0 、 4.0$ 或 $6.0 \mathrm{~g}$ 催 化剂样品, 避光摚拌 $30 \mathrm{~min}$ 达到吸附平衡, 然后超 声 $(21 \mathrm{~Hz} 、 950 \mathrm{~W}$ ) 乳化 $10 \mathrm{~min}$, 之后置于反应器中, 开启光源进行反应, 每隔一段时间间隔取样, 并将 反应液稀释 100 倍, 震荡 $24 \mathrm{~h}$ 以确保 NB 完全溶于水 中, 过滤分离, 采用高效液相色谱测试 NB 及副产物 苯胺含量。在催化剂加入量为 $4.0 \mathrm{~g}$, 其他条件不变 的条件下进行空白和悬浮体系的对照试验。

\section{6 测试与表征}

采用傅立叶变换红外光谱(FT-IR, Spectrum Two, 英国 PE 公司)和 $X$ 射线光电子能谱(XPS, Amicus Budget, 日本岛津公司)对改性粒子表面化学状态及 其电子结构进行表征。在氩气气氛下, 利用热重分析 仪(TG, NETZSCH STA 449F3, 德国耐驰公司)对改性 粒子进行热失重分析, 升温速率 $10^{\circ} \mathrm{C} / \mathrm{min}$ 。采用接触 角测定仪 (JYC-1, 上海方瑞仪器有限公司) 测定纳米 粒子接触角, 先采用 FY 手动压片机将光催化剂压成 薄片, 然后测定水滴在其表面的接触角。通过 X 射线 粉末衍射仪(XRD, D8 ADVANCE, 德国布鲁克公司) 进行物相分析。通过场发射扫描电子显微镜(FE-SEM, JSM-6700F, 日本 JEOL 公司)和高倍透射电子显微镜 (HRTEM, JEM-2100, 日本 JEOL 公司)观察样品表面形 貌及粒径大小。采用激光粒度分布仪(BT-90, 丹东百特 仪器有限公司)分析改性粒子粒径分布。

\section{2 结果与讨论}

\section{1 表面结构}

图 1 为改性前后各物质的 FT-IR 谱图, 谱线 $\mathrm{a} 、 \mathrm{~b}$ 、 c 分别对应 $\mathrm{Arg} 、 \mathrm{SA} 、 \mathrm{TiO}_{2}$ 的谱图, 谱线 d、e、f、g 分别对应改性粒子不辐照、紫外辐照 $1 \mathrm{~h} 、 3 \mathrm{~h}$ 和 $24 \mathrm{~h}$ 的谱图。(a)1627, 1562, 3099 和 $3274 \mathrm{~cm}^{-1}$ 对应 $\mathrm{NH}_{2}$-; 2944,2863 和 $1474 \mathrm{~cm}^{-1}$ 对应 $\mathrm{NH}_{3}^{+}{ }^{[23]}$; 1331 和 $1441 \mathrm{~cm}^{-1}$ 对应 COO-的对称与非对称伸缩振动峰; (b) 1464 和 $1483 \mathrm{~cm}^{-1}$ 对应苯环; 1443 和 $1611 \mathrm{~cm}^{-1}$ 对应 COO-的
对称振动峰和不对称振动峰; (c)1629 和 $3380 \mathrm{~cm}^{-1}$ 对 应 $\mathrm{O}-\mathrm{H} ; 1402 \mathrm{~cm}^{-1}$ 对应 Ti-OH; $669 \mathrm{~cm}^{-1}$ 对应 $\mathrm{Ti}-\mathrm{O}-\mathrm{Ti}^{[5]}$ 。改性光催化剂谱图(d)中新出现苯环、 $\mathrm{NH}_{2}-$ 特征峰、以及 1687 和 $1384 \mathrm{~cm}^{-1}$ 分别对应的 COOTi 基的对称振动峰和不对称振动峰, 说明 $\mathrm{SA}$ 与 $\mathrm{Arg}$ 均通过羧基成功地接枝到 $\mathrm{TiO}_{2}$ 表面。但改性后 的 $\mathrm{TiO}_{2}$ 的红外光谱中未出现 $\mathrm{NH}_{3}^{+}$-特征峰, 可归因 于氨基与 $\mathrm{TiO}_{2}$ 表面产生互动导致去质子化 ${ }^{[24]}$, 进一 步证实 $\mathrm{SA}$ 与 $\mathrm{Arg}$ 成功地接枝到 $\mathrm{TiO}_{2}$ 表面。在 1629 和 $3380 \mathrm{~cm}^{-1}$ 出现着基的特征峰, 但强度大大减弱, 说明颗粒内尚有未反应的残余羟基。经 $1 \mathrm{~h}$ 和 $3 \mathrm{~h}$ 紫 外辐照后红外谱图基本不变, 说明改性纳米光催化剂 在紫外光下有较好的稳定性; 经过 $24 \mathrm{~h}$ 连续紫外光辐 照后，苯环特征峰基本消失，而 $\mathrm{NH}_{2}$-特征峰依然明 显, 说明此时光催化剂表面的水杨酸已降解完全, 而精氨酸依然存在, 证实精氨酸改性结构更稳定。
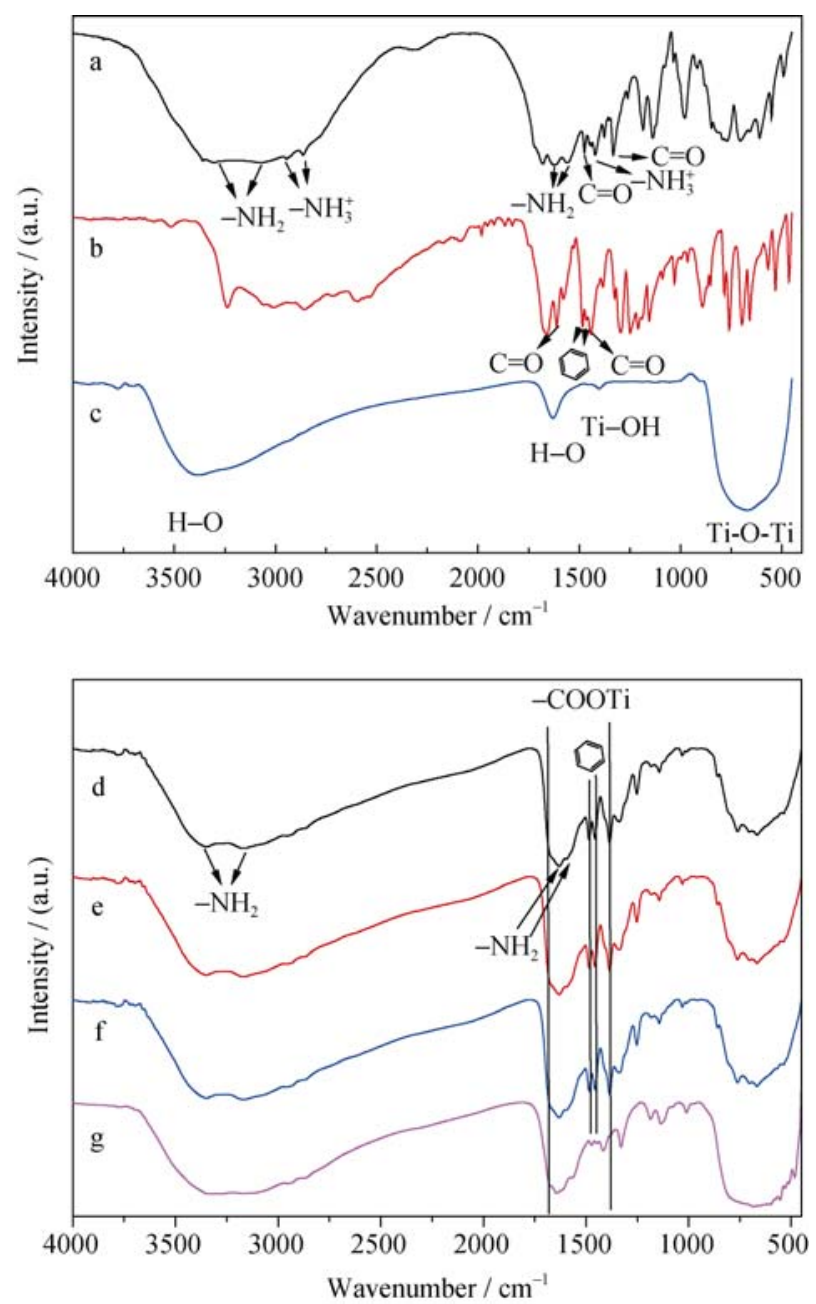

图 1 改性前后各物质的傅里叶变换红外光谱

Fig. 1 FT-IR spectra of different samples (a) Arg; (b) $\mathrm{SA}$; (c) $\mathrm{TiO}_{2}$; (d) $\mathrm{TiO}_{2}-\mathrm{SA} / \mathrm{Arg}$ before irradiation; (e) $\mathrm{TiO}_{2-}$ $\mathrm{SA} / \mathrm{Arg}$ afterlh irradiation; (f) $\mathrm{TiO}_{2}-\mathrm{SA} / \mathrm{Arg}$ after $3 \mathrm{~h}$ irradiation; (f) $\mathrm{TiO}_{2}$-SA/Arg after $24 \mathrm{~h}$ irradiation 
Nakamoto ${ }^{[27]}$ 提出可根据式 $\Delta v=v_{\mathrm{as}}-v_{\mathrm{s}}\left(v_{\mathrm{as}}\right.$ 为 $\mathrm{COO}-$ 的不对称振动峰的值; $v_{\mathrm{s}}$ 为 $\mathrm{COO}-$ 的对称振动峰的值) 判断䍨酸根离子与金属离子之间的键合形式(单齿、螯 合或桥连), 若 $\Delta v$ (改性物 $)<\Delta v$ (游离羧酸), 则键合方 式为单齿配位; 反之则为鳌合或桥连配位。由 $\Delta v\left(\mathrm{TiO}_{2}-\mathrm{SA} / \mathrm{Arg}\right)=303 \mathrm{~cm}^{-1}>\Delta v$ (游离羧酸)可以得出 $\mathrm{SA}$ 和 $\mathrm{Arg}$ 是以桥连或螯合方式键合在纳米 $\mathrm{TiO}_{2}$ 表面。

图 2 为改性后样品的 C1s、N1s 和 $\mathrm{O} 1 \mathrm{~s}$ 的 XPS 谱图, 并对样品中出现的 $\mathrm{O} 1 \mathrm{~s} 、 \mathrm{~N} 1 \mathrm{~s} 、 \mathrm{C} 1 \mathrm{~s}$ 峰进行高 斯拟合。从图 2(a)可以看出, C1s 的 XPS 中出现五重 峰, 分别对应 $\mathrm{C}-\mathrm{C}(284.9 \mathrm{eV}), \mathrm{C}-\mathrm{N}(286.9 \mathrm{eV})$, $\mathrm{C}-\mathrm{O}-\mathrm{C}$ 或 $\mathrm{C}-\mathrm{O}-\mathrm{Ti}(287.0 \mathrm{eV}), \mathrm{O}-\mathrm{C}-\mathrm{O}(288.6 \mathrm{eV})$ 和 $\mathrm{O}-\mathrm{CN}_{2}(290.6 \mathrm{eV}) ; \mathrm{N} 1 \mathrm{~s}$ 的 XPS 中出现四重峰(图 2(b)), 分别对应 $\mathrm{C}-\mathrm{NH}_{2}(397.0 \mathrm{eV}), \mathrm{C}-\mathrm{N}-\mathrm{C}(397.8 \mathrm{eV}), \mathrm{C}-\mathrm{N}$ $(403.5 \mathrm{eV})$; O1s 的 XPS 中出现三重峰(图 2(c)), 分别 对应 Ti-OH 或 Ti-O-C(532.1 eV), COO-(534.1 eV), $\mathrm{H}_{2} \mathrm{O}(535.5 \mathrm{eV})$ 。

FT-IR 和 XPS 的结果符合 $\mathrm{Li}$ 等 ${ }^{[22]}$ 提出的 SA 与 二氧化钛表面形成稳定的六元环结构和 Cropek 等 ${ }^{[24]}$ 提出 Arg 与二氧化钛表面形成的桥连结构。目前, 金 属氧化物表面与有机基体作用机理主要有可变形层 理论、约束层理论、化学键理论和浸润效应理论。 $\mathrm{SA}$ 与 $\mathrm{Arg}$ 改性过程有新化学键产生, 反应过程符 合化学键理论, 具体反应过程步骤可分为四步 ${ }^{[28]}$ : (1)二氧化钛表面羟基质子化; (2)一部分质子化羟基 与 $\mathrm{SA}$ 上的羒基形成类似酯键; (3)一部分质子化差基 与改性剂中的羟基形成氢键桥连; (4)伴随脱水氢键 逐渐转化为共价键, 并形成稳定的环状螯合结构或 桥连结构。

\section{2 热稳定性}

采用 $\mathrm{TG}$ 技术对光催化剂的热稳定性进行评价, 结果如图 3 所示。由图 3 可见, 改性后 $\mathrm{TiO}_{2}$ 的重量 损失是由表面 SA 与 $\mathrm{Arg}$ 分解导致的。在 $130^{\circ} \mathrm{C}$ 以下 的重量损失归因于吸收水分的挥发; 在 $130 \sim 210^{\circ} \mathrm{C}$ 的 失重归因于表面的 SA 的热分解 ${ }^{[5]}$, 重量损失率约为 $0.7 \%$; 在 $210 \sim 250^{\circ} \mathrm{C}$ 的失重归因于 $\mathrm{SA}$ 与 $\mathrm{Arg}$ 共同热 分解，重量损失率约为 $0.3 \%$; 在 $250 \sim 500{ }^{\circ} \mathrm{C}$ 失重归 因于 $\operatorname{Arg}$ 的热分解, 重量损失率约为 $4.0 \%$ 。 SA 和 $\operatorname{Arg}$ 的总重量损失率约为 $5.0 \%$, 说明 $\operatorname{Arg}$ 接枝量相对 较大。在紫外光辐照 $1 \mathrm{~h}$ 与 $3 \mathrm{~h}$ 后的热重曲线显示重 量损失基本与辐照前一致, 进一步证实改性纳米光 催化剂在紫外光下有较好的稳定性。

\section{3 粒径分布}

采用激光粒度分布仪对改性前后光催化剂在水 中的粒度分布进行分析。改性前纳米粒子在水中团
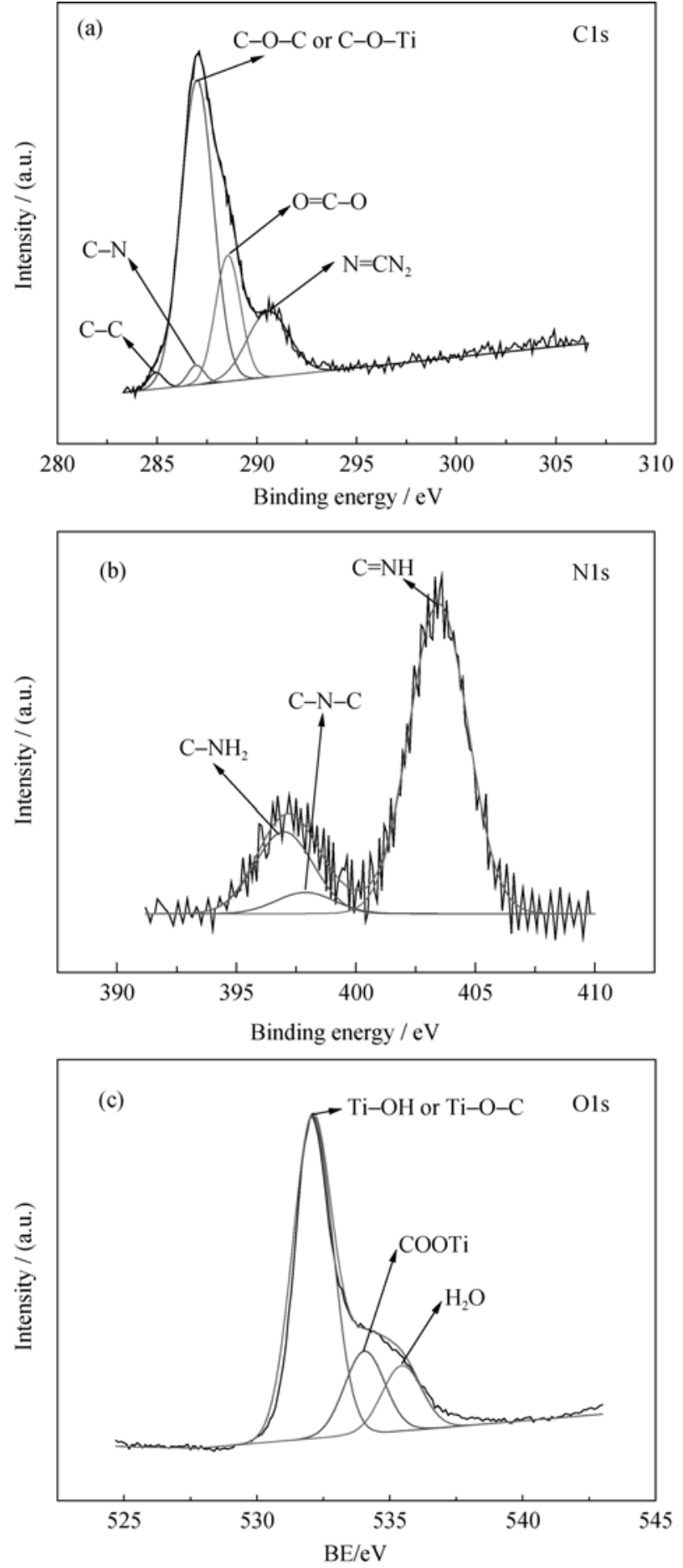

图 2 改性后样品的 C1s (a)、N1s (b) 和 O1s (c)的 XPS 谱图 Fig. 2 C1s (a), N1s (b) and O1s (c) XPS spectra of modified samples

聚现象较严重, 平均粒径为 $183 \mathrm{~nm}$ 且粒度分布范围 较宽, 多分散指数(PDI, polydisperse index)为 0.713 。 经 SA 和 $\mathrm{Arg}$ 共改性后, 平均粒径变为 $159 \mathrm{~nm}$, 而多 分散指数降为 0.631 , 说明改性后固体颗粒在水中的 分散情况改善, 团聚减弱且多分散性降低。

\section{4 接触角}

采用水滴角表征光催化剂的表面亲水性质, 如 图 4 所示, $\mathrm{TiO}_{2}$ 表面水/空气界面接触角约为 $13.9^{\circ}$; 


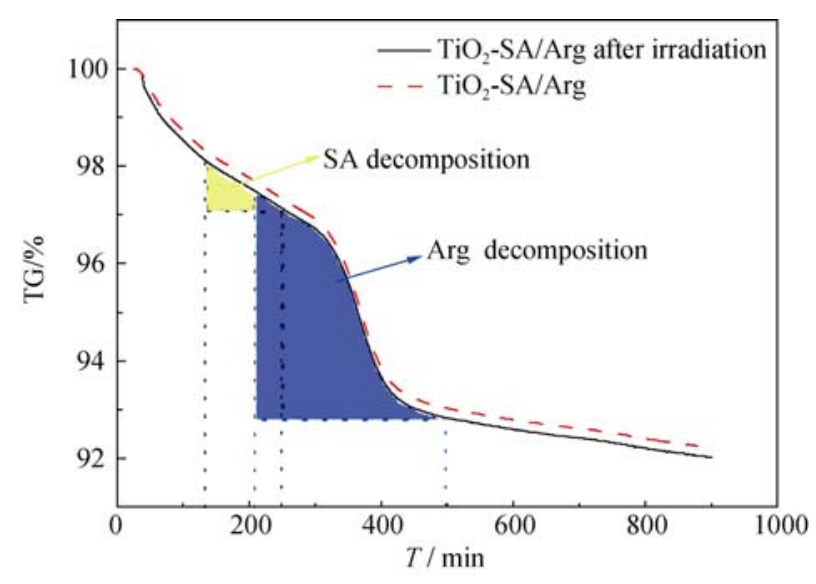

图 3 改性后样品的 TG 曲线

Fig. 3 TG curve of samples after modification

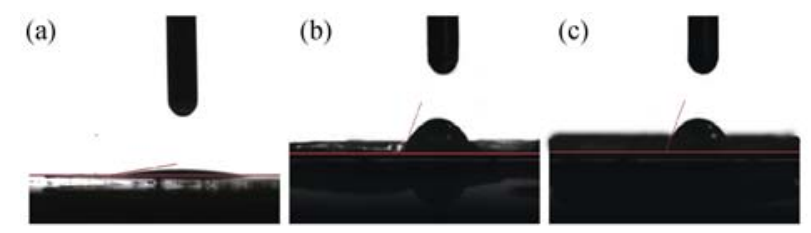

图 4 光催化剂的接触角

Fig. 4 Contact angles of photocatalyst (a) $\mathrm{TiO}_{2}$; (b) $\mathrm{TiO}_{2}-\mathrm{SA} / \mathrm{Arg}$; (c) $\mathrm{TiO}_{2}-\mathrm{SA} /$ Arg after irradiation
改性后 $\mathrm{TiO}_{2}$ 表面水/空气界面接触角约为 $79.9^{\circ}$; 经紫 外光辐照 $3 \mathrm{~h}$ 并改性后 $\mathrm{TiO}_{2}$ 表面水/空气界面接触角约 为 $78.3^{\circ}$ 。通常水/空气界面接触角可直接反映颗粒表 面的亲水性, 未改性的 $\mathrm{TiO}_{2}$ 亲水性极强, 颗粒易分散 于水相中，不易形成稳定的 Pickering 乳液; 表面改性 后的 $\mathrm{TiO}_{2}$ 亲水性减弱, 在油一水界面的吸附能力增强; $3 \mathrm{~h}$ 紫外光辐照后改性 $\mathrm{TiO}_{2}$ 表面亲水性基本不变, 说 明紫外辐照对光改性 $\mathrm{TiO}_{2}$ 表面性质影响不明显。

\section{5 形貌及光响应范围}

采用扫描电子显微镜及高倍透射电子显微镜对 光催化剂的形貌进行表征, 如图 5 所示。从图 5 可以 看出, 改性前纳米粒子团聚严重, 粒径约为 $30 \mathrm{~nm}$; 改性后纳米粒子粒径增大到 $35 \mathrm{~nm}$; 经过 $3 \mathrm{~h}$ 紫外光 辐照后纳米粒子粒径及形貌基本不变。由 $\mathrm{TiO}_{2}$ 和 $\mathrm{TiO}_{2}-\mathrm{SA} / \mathrm{Arg}$ 的 HRTEM 照片中可见, 光催化剂样品 均呈不规则的片状纳米粒子; 改性后的光催化剂颗 粒间的分界更明显, 说明改性后表面有机层起到了 防止团聚的作用。改性前后，锐钛矿相(101)和金红石 相(101)晶面间距分别为 0.350 和 $0.249 \mathrm{~nm}$, 表明共改 性后的 $\mathrm{TiO}_{2}$ 晶相结构变化不明显。此外, 从图 5 可 以看到改性光催化剂表面的有机层结构。
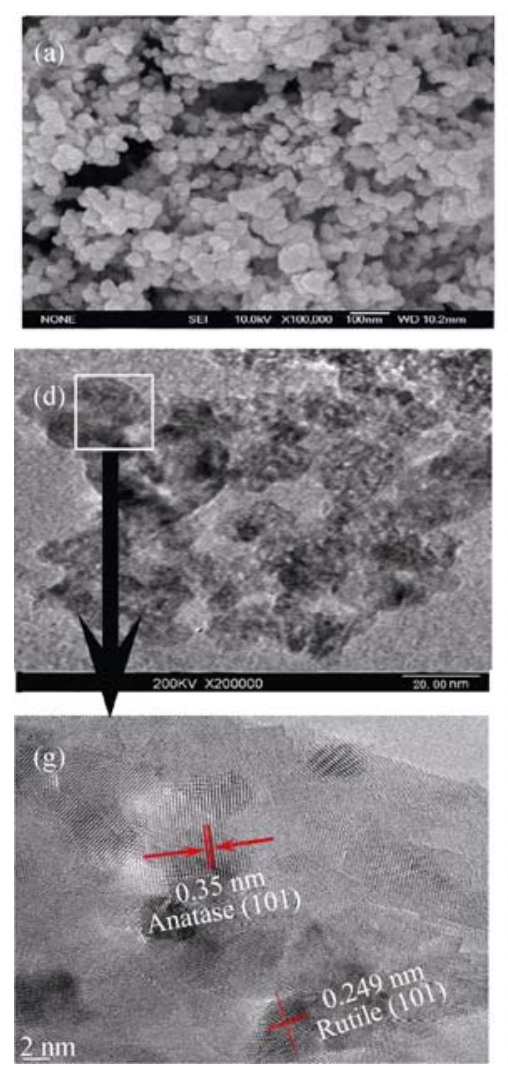
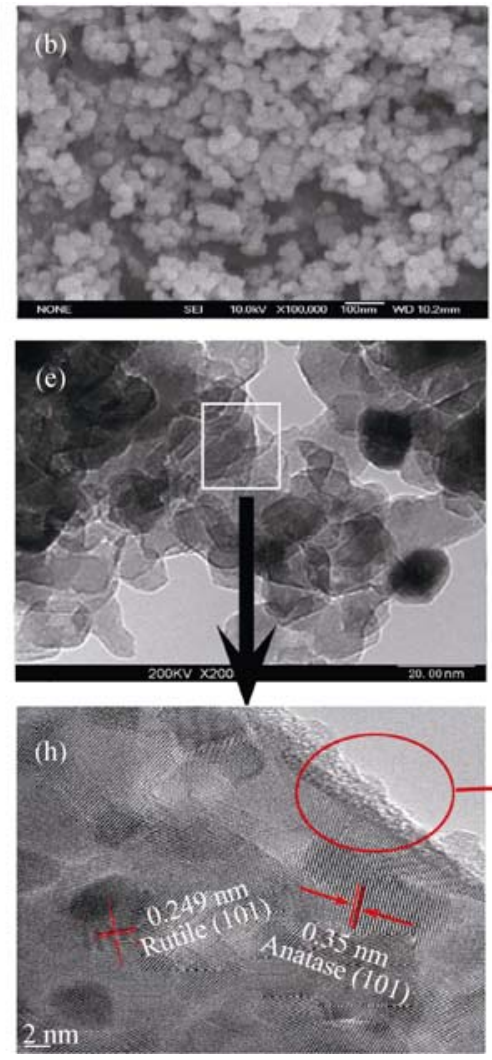
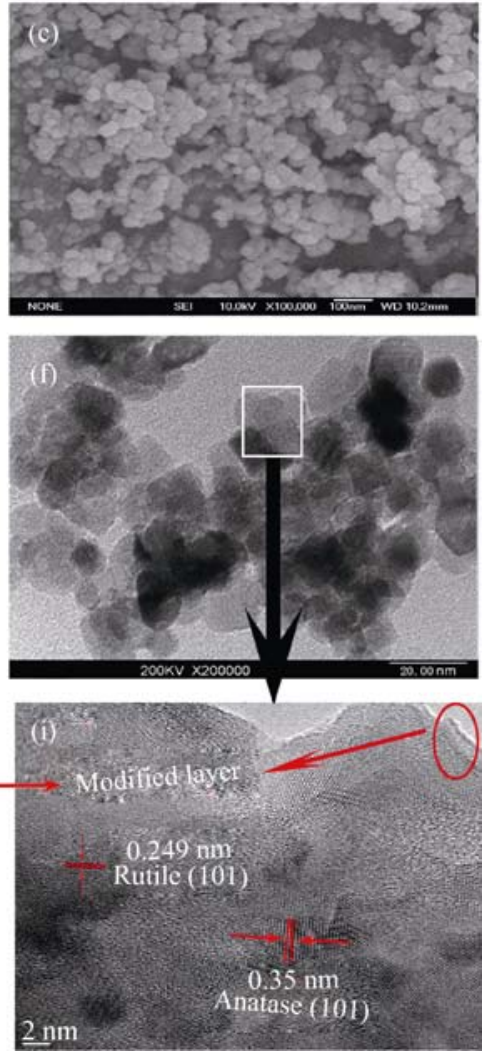

图 5 光催化剂的扫描和高倍透射电子显微镜照片

Fig. 5 SEM and HRTEM images of photocatalysts (a,d,g) $\mathrm{TiO}_{2}$; (b,e,h) $\mathrm{TiO}_{2}$-SA/Arg; (c,f,i) $\mathrm{TiO}_{2}$-SA/Arg after irradiation 
改性粒子的紫外-可见漫反射光谱，如图 6 所 示。纯 $\mathrm{TiO}_{2}$ 只在紫外区有较强吸收, 吸收阈值为 $415 \mathrm{~nm}$; 而改性颗粒的吸收光谱发生了较大程度 的红移，在可见光区有一定的吸收，吸收阈值在 $600 \mathrm{~nm}$ 以上，比文献[28]的 $\mathrm{SA}$ 改性 $\mathrm{TiO}_{2}$ 的吸收阈 值(500 nm) 有了进一步提高, 说明两种物质共改性 使得光催化剂的可见光吸收能力进一步提高。

\section{6 对硝基苯的吸附能力}

采用等温吸附曲线分析催化剂对 NB 的吸附能 力, 结果如图 7 所示, $\mathrm{SA}$ 和 $\mathrm{Arg}$ 对 $\mathrm{TiO}_{2}$ 的表面改性 提高了光催化剂对 NB 的吸附能力, 催化剂对 NB 的 饱和吸附容量提高约一倍, 这是由于改性颗粒表面 官能团如苯环、氨基等与 NB 分子产生氢键、 $\mathrm{n}-\pi$ 和 $\pi-\pi$ 等相互作用，导致对 NB 的吸附作用增强 ${ }^{[22]}$ 。光 催化剂对 NB 的吸附能力的增强使得 NB 到光催化剂 表面的整体迁移速率加快。

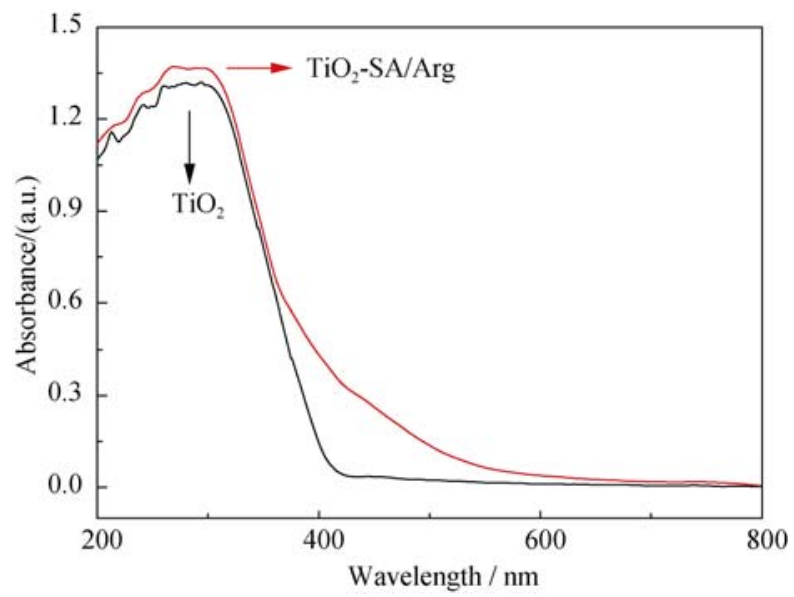

图 6 样品的紫外-可见光漫反射光谱

Fig. 6 UV-Vis diffuse reflectance spectra of samples

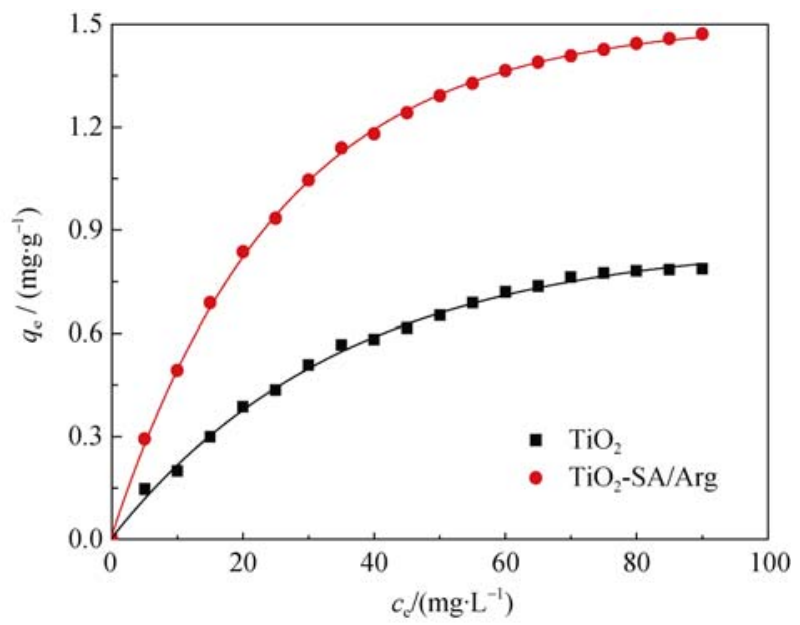

图 7 水溶液中硝基苯的吸附曲线

Fig. 7 Adsorption isotherms of NB measured in aqueous solutions

\section{7 在油一水界面的吸附性能}

Pickering 乳液体系降解 NB 过程中, 光催化剂颗 粒在油-水界面的吸附能力决定了乳液的稳定性和 界面催化体系的催化效率。不同油水体积比条件下 乳液体积比随时间的关系曲线如图 8 所示。由图 8 可见，改性颗粒在水-油界面吸附性能随油水比的提 高而增强，乳液直径逐渐变大。从分层位置可以看出， 改性粒子稳定的乳液水相和油相均出现分层，说明 改性颗粒的亲水亲油性适中。总体来说, $7 \mathrm{~h}$ 静置后 Pickering 乳液的乳状液部分仍占有较大体积分数 $(70 \% \sim 80 \%)$, 改性颗粒在油一水界面吸附能力较强。

\section{8 改性粒子稳定的乳液光催化过程}

为了研究改性催化剂稳定的 Pickering 乳液对高 浓度废水的降解效果, 进行了 NB 降解实验, 结果如 图 9 所示。由 NB 直接光降解和逸散, 空白实验中 NB 大约有 $8.0 \%$ 的降解率。在悬浮体系中, $\mathrm{TiO}_{2}$ 与 $\mathrm{TiO}_{2}-\mathrm{SA} / \mathrm{Arg}$ 对应的降解率分别为 $28.0 \%$ 与 $63.7 \%$, 降 解率都较低, 主要是由于亲水性 $\mathrm{TiO}_{2}$ 主要分散在水相 中，只能降解溶于水相中的少量 $\mathrm{NB}$, 而改性颗粒虽 然吸附于油-水界面，但整体界面面积较小，催化效率 较低。体系乳化后，随着反应界面面积进一步增加， 反应降解效率也进一步提高。当固体粒子质量分数分 别为 $2.66 \% 、 5.18 \% 、 9.85 \%$ 时, Pickering 乳液降解效 率可达 $82.2 \%$ 、99.2\%和 $99.4 \%$ 。当 Pickering 乳液体 系固体粒子用量较低时，在界面形成的粒子膜较薄， 在反应过程中，液滴相互碰撞易发生聚并，导致油滴 尺寸变大和界面面积变小，反应后期降解效率变差; 增加固体粒子用量可使固体粒子膜变厚, 乳液的稳定 性增强，降解反应得以快速进行; 但当固体粒子浓度 过高时, 会导致体系浊度增大, 体系透光性能力变差, 导致反应只在表层发生，从而降低降解效率。

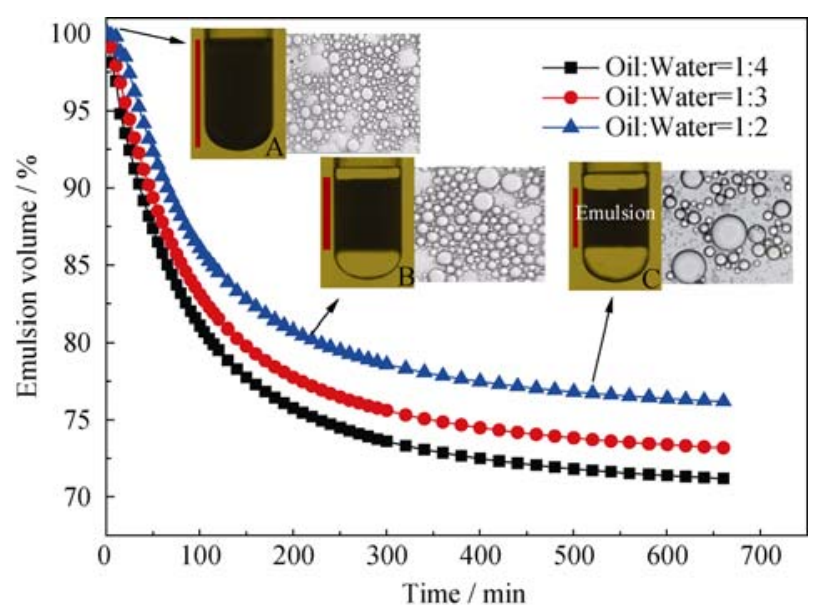

图 $8 \quad \mathrm{TiO}_{2}-\mathrm{SA} / \mathrm{Arg}$ 稳定的 Pickeing 乳液的稳定性

Fig. 8 Stability of Pickeing emulsion stabilized by $\mathrm{TiO}_{2}-$ $\mathrm{SA} / \mathrm{Arg}$ 
图 9 中 HPLC 曲线给出了乳液体系中苯胺及硝 基苯特征峰变化情况, 苯胺的峰面积先增大后减小, 即体系中苯胺含量先增加后减少, $\mathrm{TiO}_{2}-\mathrm{SA} / \mathrm{Arg}$ 颗粒 对 $\mathrm{NB}$ 的降解反应前期与 $\mathrm{TiO}_{2}-\mathrm{Arg}$ 相似, 具有还原过 程特点; 后期反应又具有 $\mathrm{TiO}_{2}$ - $\mathrm{SA}$ 的氧化过程特点。 这是由于 Arg 具有很强的给电子特性, 可以作为空 穴陷阱而防止电子-空穴复合, 产生光生电子可将 $\mathrm{NB}$ 还原为苯胺, 但没有进一步降解苯胺的能力; 但 $\mathrm{SA}$ 与 $\mathrm{TiO}_{2}$ 形成的 $\pi-\pi$ 共轭结构却不能有效防止电 子-空穴复合, 反应过程依旧遵循氧化途径 ${ }^{[1,23]}$ 。反 应前期由于 $A r g$ 吸附量较大, 还原反应显著; 反应后 期苯胺继续还原，氧化反应变显著。

实验研究发现共改性后的 $\mathrm{TiO}_{2}$ 稳定的 Pickering 乳液具有良好的光催化活性, 主要原因是 ${ }^{[21,23]}$ : (1)光 催化剂表面的 SA 和 Arg 具有很好的电子转移特性, 使催化剂在紫外或近紫外的光吸收能力增强; (2)Arg 和 $\mathrm{SA}$ 表面改性增强了 $\mathrm{TiO}_{2}$ 表面与 $\mathrm{NB}$ 的耦合和吸附; (3)Arg 强给电子性改性使得 NB 降解过程存在还原途 径; (4)改性催化剂表面具有亲油链和亲水性基团, 类 似于乳化剂分子将目标物完全包裹, 增加了催化剂与 目标物的接触面积, 反应活性位点利用率较高。

借鉴文献提出的硝基苯降解反应过程 ${ }^{[25]}$, 提出 Pickering 乳液体系中具体反应过程如图 10 所示: (1) 由于水相透光性较好, 水相一侧改性粒子首先受到 光激发产生光生电子, 进而产生电子-空穴对, $\mathrm{TiO}_{2}$ 表面吸附 Arg 的 NB 分子被还原为苯胺。(2)反应逐 渐转移到油相内部, 中间产物苯胺受到强氧化性自 由基攻击继续分解, 产生的小分子物质从油相转移 至水相, 降解反应得以快速进行。

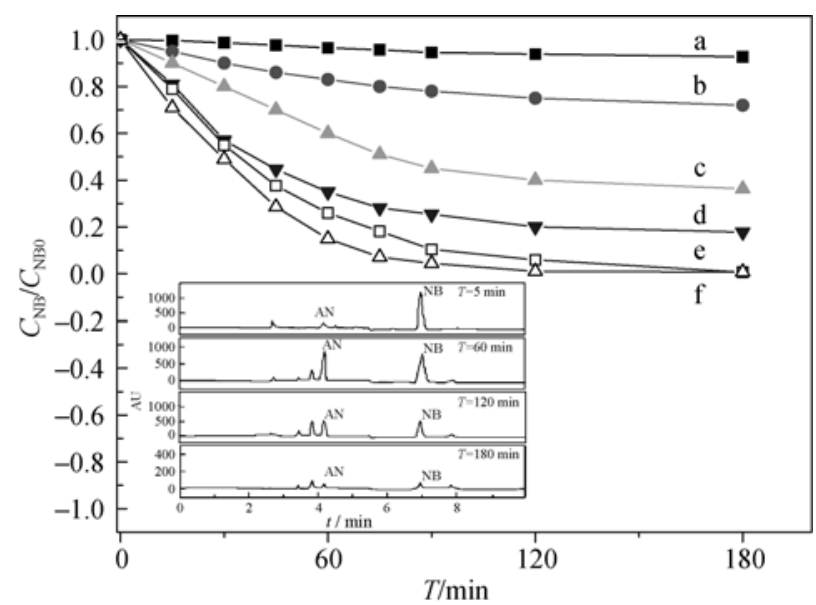

图 9 光催化降解 NB 曲线图

Fig. 9 Curves of photocatalytic degradation of NB

(a) Blank; (b) $\mathrm{TiO}_{2}$ in suspension; (c) $\mathrm{TiO}_{2}-\mathrm{SA} / \mathrm{Arg}$ in suspension; (d) $2.66 \mathrm{wt} \% \mathrm{TiO}_{2}-\mathrm{SA} / \mathrm{Arg}$ in emulsion; (e) $9.85 \mathrm{wt} \% \mathrm{TiO}_{2}-\mathrm{SA} / \mathrm{Arg}$ in emulsion; (f) $5.18 \mathrm{wt} \% \mathrm{TiO}_{2}-\mathrm{SA} / \mathrm{Arg}$ in emulsion. Inset is the HPLC curves of emulsion

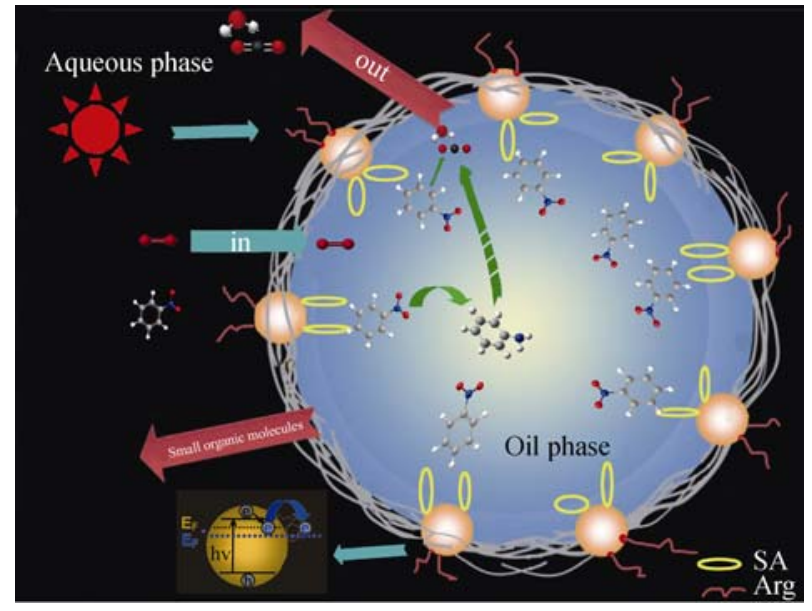

图 10 水杨酸和精氨酸共改性 $\mathrm{TiO}_{2}$ 颗粒在水/油界面自组装 形成的高效皮克林乳液光催化体系

Fig.10 Schematic of a highly efficient Pickering emulsionbased photocatalytic system formed by self-assembling $\mathrm{TiO}_{2}$ particles co-modified by salicylic acid and arginine at water/oil interface

\section{3 结论}

采用水杨酸和精氨酸对纳米 $\mathrm{TiO}_{2}$ 进行表面改性 成功制备了 $\mathrm{TiO}_{2}-\mathrm{SA} / \mathrm{Arg}$ 共改性纳米颗粒, 并将其稳 定的 Pickering 乳液体系用于 NB 的光催化降解。

1) 与未改性粒子相比，改性粒子表面亲水性减 弱, 在水中的分散性变好, $3 \mathrm{~h}$ 紫外光照射后表面有 机层未发生明显的自降解。

2) SA 以六元环的鳌合形式、Arg 以桥连形式与 $\mathrm{TiO}_{2}$ 相连。

3) Pickering 乳液体系可显著提高硝基苯的降解 速率。3 h 降解实验后, 硝基苯的降解率可达 $99.4 \%$, 且降解过程具有还原反应的特点。

\section{参考文献:}

[1] WANG BIN, ZHANG GUANG-XIN, ZHENG SHUI-LIN, et al. Effect of calcination temperature on crystal structure and photocatalytic property of $\mathrm{TiO}_{2} /$ diatomite nanoparticles. Journal of Inorganic Materials, 2014, 29(4): 382-386.

[2] LIU GUO-CONG, LI HAI-BIN, DONG HUI. Ultrasonic-hydrothermal synthesis and photocatalytic activities of La-doped mesoporous $\mathrm{TiO}_{2}$ microspheres. Journal of Inorganic Materials, 2011, 26(7): 739-746.

[3] ZHANG QING-HONG. Progress on $\mathrm{TiO}_{2}$-based nanomaterials and Its utilization in the clean energy technology. Journal of Inorganic Materials, 2012, 27(1): 1-10.

[4] HUGO I D L, BENITO S R. Photocatalytic Technologies. Beijing: 
Science Press, 2010: ix-xii.

[5] NSIB M F, MAAYOUFI A, MOUSSA N J, et al. $\mathrm{TiO}_{2}$ modified by salicylic acid as a photocatalyst for the degradation of monochlorobenzene via Pickering emulsion way. Photochem. Photobiol. A: Chem., 2013, 251: 10-17.

[6] RAMSDEN W. Separation of solids in the surface-layers of solutions and suspensions. Proc. R. Soc. Lon., 1903-1904, 72: 156-164.

[7] PICKERING S U. Emulsions. J. Am. Chem. Soc., 1907, 91: 20012021.

[8] LAGALY G, REESE M, ABEND S. Smectites as colloidal stabilizers of emulsions II: Rheological properties of smectite-laden emulsions. Appl. Clay. Sci. 1999, 14: 279-298.

[9] TARIMALA S, DAI L L. Structure of microparticles in solidstabilized emulsions. Langmuir, 2004, 20: 3490-3494.

[10] BINKS B P. Particles as surfactants-similarities and differences Curr. Opin. Colloid Interface Sci., 2002, 7: 21-41.

[11] YANG F, WANG J, LAN Q, et al. Research progress on Pickering emulsions. Prog. Chem., 2009, 21(7): 1418-1426.

[12] WU WEI, GAO SHUANG, TU WEI-XIA, et al. Intensified photocatalytic degradation of nitrobenzene by Pickering emulsion of $\mathrm{ZnO}$ nanoparticles. Particuology, 2010, 8(5): 453-457.

[13] NAKATO T, UEDA H, HASHIMOTO S, et al. Pickering emulsions prepared by layered niobate $\mathrm{K}_{4} \mathrm{Nb}_{6} \mathrm{O}_{17}$ intercalated with organic cations and photocatalytic dye decomposition in the emulsions. ACS Appl. Mater. Interfaces, 2012, 4(8): 4338-4347.

[14] ZHAI WAN-YING, LI GAI-PING, YU PING, et al. Silver phosphate/carbon nanotube-stabilized Pickering emulsion for highly efficient photocatalysis. J. Phys. Chem. C, 2013, 117: 15183-15191.

[15] CLARIZIA L, SPASIANO D, DI SOMMA I, et al. Copper modified$\mathrm{TiO}_{2}$ catalysts for hydrogen generation through photoreforming of organics. a short review. Int. J. Hydrogen Energ., 2014, 39(30): 16812-16831.

[16] LÜ YU-ZHEN, ZHANG SHENG-NAN, DU YUE-FAN, et al. Effect of oleic acid surface modification on dispersibility of $\mathrm{TiO}_{2}$ nanoparticles in transformer oils. Journal of Inorganic Materials, 2013, 28(6): 594-598.

[17] YAO CHAO, GAO GUO-SHENG, LIN XI-PING, et al. Surface modification of nanosized $\mathrm{TiO}_{2}$ with silane coupling reagent. Journal of Inorganic Materials, 2006, 21(2): 315-321.
[18] TAI HUI-LING, JIANG YA-DONG , XIE GUANG-ZHONG, et al. Fabrication and gas sensitivity study of polyprrole/titanium oxide composite thin films. Journal of Inorganic Materials, 2007, 22(3): 524-528.

[19] RAZA M, BACHINGER A, ZAHN N, et al. Interaction and UV-stability of various organic capping agents on the surface of anatase nanoparticles. Materials, 2014, 7: 2890-2912.

[20] WANG NAN, ZHU LI-HUA, DENG KE-JIAN, et al. Visible light photocatalytic reduction of $\mathrm{Cr}(\mathrm{VI})$ on $\mathrm{TiO}_{2}$ in situ modified with small molecular weight organic acids. Appl. Catal. B-Environ., 2010, 95: 400-407.

[21] DOBSON K D, MCQUILLAN A J. In situ infrared spectroscopic analysis of the adsorption of aliphatic carboxylic acids to $\mathrm{TiO}_{2}$, $\mathrm{ZrO}_{2}, \mathrm{Al}_{2} \mathrm{O}_{3}$, and $\mathrm{Ta}_{2} \mathrm{O}_{5}$ from aqueous solutions. Spectrochim. Acta A, 1999, 55: 1395-1405.

[22] LI SHUN-XING, ZHENG FENG-YING, CAI SHU-JIE, et al. A visible light assisted photocatalytic system for determination of chemical oxygen demand using 5 -sulfosalicylic acid in situ surface modified titanium dioxide. Sensor. Actuat. B-Chem., 2013, 188: 280-285.

[23] LI SUNG-XING, ZHENG FENG-YING, CAI WEN-LIAN, et al. Surface modification of nanometer size $\mathrm{TiO}_{2}$ with salicylic acid for photocatalytic degradation of 4-nitrophenol. J. Hazard. Mater., 2008, B135: 431-436.

[24] CROPEK D, KEMME P A, MAKAROVA O V, et al. Selective photocatalytic decomposition of nitrobenzene using surface modified $\mathrm{TiO}_{2}$ nanoparticles. J. Phys. Chem. C, 2008, 112: 8311-8318.

[25] HUANG H Y, ZHOU J H, LIU H L, et al. Selective photoreduction of nitrobenzene to aniline on $\mathrm{TiO}_{2}$ nanoparticles modified with amino acid. J. Hazard. Mater., 2010, 178: 994-998.

[26] NAKAYAMA N, HAYASHI T. Preparation of $\mathrm{TiO}_{2}$ nanoparticles surface-modified by both carboxylic acid and amine: dispersibility and stabilization in organic solvents. Colloid Surf. A-Physicochem. Eng. Asp., 2008: 317(1/2/3): 543-550.

[27] NAKAMOTO K. Infrared and Raman Spectra of Inorganic and Coordination Compounds. New York: Wiley, 1978: 232-233.

[28] ZHANG QIAO-LING, LI LEI, LIU YOU-ZHI, et al. Grafting dynamics, structures and properties of nano $\mathrm{TiO}_{2}$-SA photocatalytic materials. Acta Phys. -Chim. Sin., 2015, 31(6): 1015-1024. 